\title{
An apparatus for immersing trapped ions into an ultracold gas of neutral atoms
}

\author{
Stefan Schmid, ${ }^{1,2}$ Arne Härter, ${ }^{1,2}$ Albert Frisch, ${ }^{2}$ Sascha Hoinka, ${ }^{2,}$ (a) and Johannes Hecker Denschlag ${ }^{1,2}$ \\ ${ }^{1)}$ Institut für Quantenmaterie und Center for Integrated Quantum Science and Technology IQST, Universität Ulm, 89069 Ulm, \\ Germany \\ ${ }^{2)}$ Institut für Experimentalphysik und Zentrum für Quantenphysik, Universität Innsbruck, 6020 Innsbruck, \\ Austria
}

\begin{abstract}
We describe a hybrid vacuum system in which a single ion or a well defined small number of trapped ions (in our case $\mathrm{Ba}^{+}$or $\mathrm{Rb}^{+}$) can be immersed into a cloud of ultracold neutral atoms (in our case $\mathrm{Rb}$ ). This apparatus allows for the study of collisions and interactions between atoms and ions in the ultracold regime. Our setup is a combination of a Bose-Einstein condensation (BEC) apparatus and a linear Paul trap. The main design feature of the apparatus is to first separate the production locations for the ion and the ultracold atoms and then to bring the two species together. This scheme has advantages in terms of stability and available access to the region where the atom-ion collision experiments are carried out. The ion and the atoms are brought together using a moving 1-dimensional optical lattice transport which vertically lifts the atomic sample over a distance of $30 \mathrm{~cm}$ from its production chamber into the center of the Paul trap in another chamber. We present techniques to detect and control the relative position between the ion and the atom cloud.
\end{abstract}

\section{INTRODUCTION}

In recent years, both the fields of cold trapped ions and of neutral, ultracold atomic gases have experienced an astonishing development. Full control has been gained over the respective systems down to the quantum level. Single ions can be selectively addressed and their quantum states can be coherently manipulated and read out 1 . The collective behavior of neutral atomic quantum gases can be mastered by controlling the particle-particle interactions, temperature, and physical environment ${ }^{2}$.

Over the last two decades increasing efforts have been made to study cold collisions between ions and neutral particles. One approach is to study collisions in a cold He buffer gas (see for example. ${ }^{3-6}$ ) Another approach for collisions in the regime of a few $\mathrm{K}$ uses neutral, velocity-selected particles from a beam of molecules to collide with trapped ions $\frac{7}{-}$ In recent years collisions between atoms in a magneto-optical trap (MOT) and trapped ions trap have been observed. ${ }^{8-13}$ In 2010 , in parallel to the group of M. Köh1 ${ }^{14}-16$, our group has finally demonstrated immersing trapped ions in a BEC of $\mathrm{Rb}$ atoms at $\mathrm{nK}$ temperatures. 17

Here we describe the hybrid apparatus used for our atomion collision experiments in detail. A central design concept of our setup is the spatial separation of the BEC apparatus where the ultracold atoms (or a BEC) are produced - from the ion-trapping region, where the atom-ion collision experiments are performed. This way we gain valuable optical access to the atom-ion interaction region, that is necessary for trapping, manipulating, and detecting the atoms and ions. Furthermore, the separation and isolation of the production sites ensures that any mutual disturbance between the radiofrequency (rf) Paul trap and the rf used for forced evaporative cooling of

\footnotetext{
a) Present address:ARC Centre of Excellence for Quantum-Atom Optics, Centre for Atom Optics and Ultrafast Spectroscopy, Swinburne University of Technology, Melbourne 3122, Australia
}

the atomic sample is minimized. To transport the atoms over $30 \mathrm{~cm}$ from their place of production to the trapped ions, we employ a moving 1-dimensional (1-d) optical lattice.

As demonstrated in a first set of experiments ${ }^{17}$ the apparatus enables us to study elastic and inelastic atom-ion collisions in the ultracold regime. ${ }^{18,19}$ We plan to investigate cold chemical reactions and the controlled formation of cold molecular ions, topics which recently gained considerable interest (see for example $\left.{ }^{20,21}\right)$. Furthermore, the apparatus allows for carrying out other interesting lines of research. There are proposals to study the dynamics of charged impurities in a quantum degenerate gas, $22-24$ charge transport in a gas in the ultracold domain, ${ }^{25}$ or the formation of a mesoscopic molecular ion ${ }^{26}$

The article is organized as follows: Section II describes the layout of the multi-chamber vacuum apparatus. In Sec. III, the design and the operation of the ion trap are discussed. Section IV addresses the preparation of the ultracold atom cloud in the BEC apparatus and its optical transport into the science chamber, in which the ion trap is located. In particular, we describe an experimental procedure based on atom-ion collisions used to precisely position the atom cloud with respect to the ion.

\section{THE VACUUM APPARATUS}

The vacuum apparatus consists of three main building blocks. A MOT chamber for trapping and laser cooling Rb atoms. A BEC chamber for evaporative cooling of the Rb atoms. A "science chamber" that houses the ion trap and where the ion-atom collision experiments take place. The apparatus has two floors: MOT chamber and BEC chamber form the lower section. The science chamber, forming the upper section, is located $30 \mathrm{~cm}$ above the BEC chamber (see Figure 1 and 2). The three vacuum chambers are connected via two differential pumping stages, each consisting of a differential pumping tube as well as a gate valve. If necessary, the vacuum chambers can be separated from each other by closing the gate valves. A series of vacuum gauges, pumps, and valves 


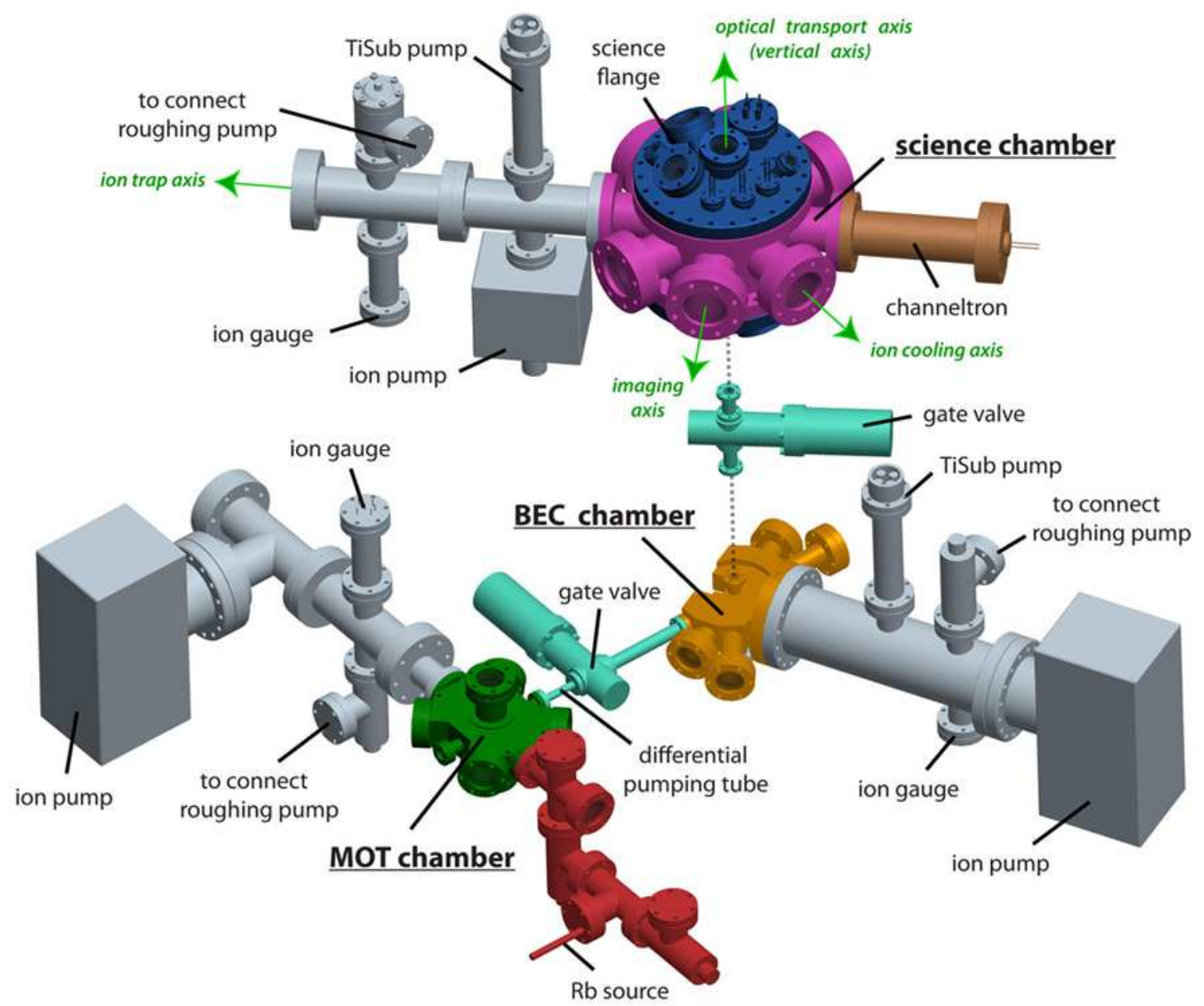

FIG. 1. Layout of the vacuum apparatus in a partially exploded view: The science chamber (upper section) is connected to the BEC chamber (in the lower section) via a differential pumping stage (turquoise) along the dashed vertical axis. The MOT chamber (green) and the BEC chamber (red) which form the lower section are also connected via a differential pumping stage (turquoise). The science chamber exhibits a large DN200CF flange (blue) on top, the "science flange", onto which the ion trap (not shown here) is mounted. A channeltron detector (brown) is connected along the axis of the linear ion trap. All three chambers are evacuated by their own pumping sections (grey). (Note: For better visibility the upper section is rotated clockwise by $90^{\circ}$ around the dashed vertical axis.)

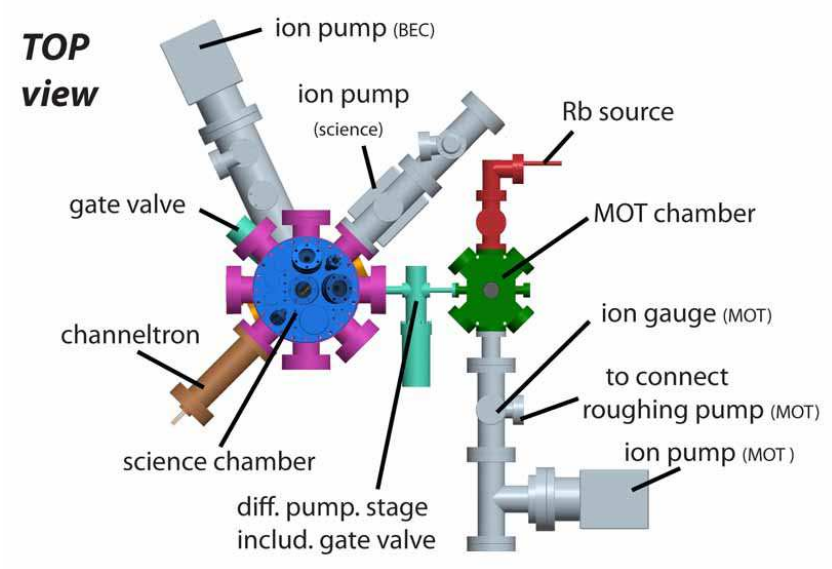

FIG. 2. Top view of the vacuum system: The science chamber (red), covers up the BEC chamber (not visible). is used to evacuate the system and to determine the pressure. By baking out the setup at temperatures between $180^{\circ} \mathrm{C}$ and $250^{\circ} \mathrm{C}$, ultrahigh vacuum (UHV) conditions are established in all three chambers. When in operation, the pressures are approximately $10^{-9}$ mbar in the MOT chamber and $10^{-11} \mathrm{mbar}$ in the BEC chamber and the science chamber.

\section{A. Lower section: BEC apparatus}

The stainless steel (AISI 316L) MOT chamber features ten optical viewports, which are required to implement the MOT laser beams, to connect to the $\mathrm{Rb}$ vapor source, to pump the chamber and to move the atoms out of the MOT chamber towards the BEC chamber. An ion getter pump 27 is used to keep the MOT chamber at UHV conditions, as measured by an UHV pressure gauge (Bayard Alpert type) ${ }^{28}$

The $R b$ vapor source is an ampule filled with bulk $R b$ and 


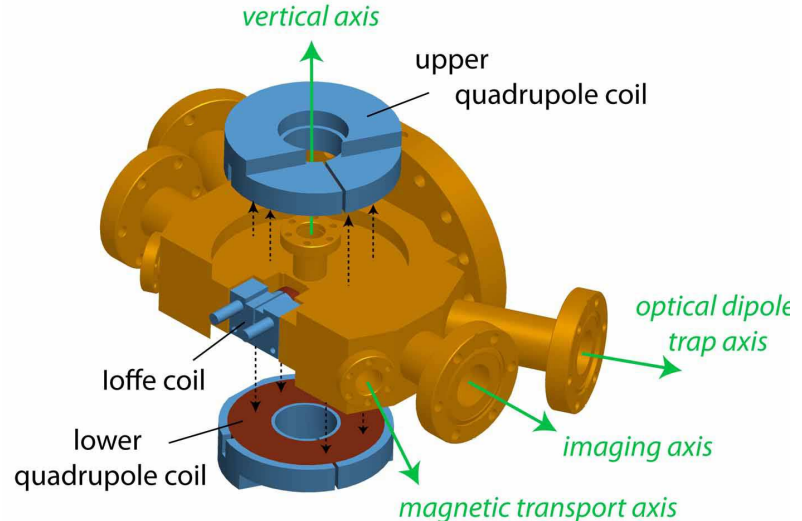

FIG. 3. Explosion view of the BEC chamber and the magnetic QUIC trap coils. The QUIC trap is generated by the two quadrupole coils and the Ioffe coil (blue). The coils are mounted outside the vacuum to the walls of the BEC chamber. The atoms enter the chamber along the magnetic transport axis and leave it along the vertical axis.

$\mathrm{He}$ as an inert $\operatorname{gas}^{29}$. Since $\mathrm{Rb}$ is very reactive when exposed to air, the ampule is not cracked until the surrounding "oven section" has been evacuated. Once Rb has been released from the ampule, the pressure in the oven section is determined by the $\mathrm{Rb}$ vapor pressure, which is $4 \times 10^{-7} \mathrm{mbar}$ at room temperature. As a consequence, the pressure in the center of the MOT chamber increases from its original value of $10^{-11} \mathrm{mbar}$ to a few times $10^{-9} \mathrm{mbar}$ and is then completely dominated by the $\mathrm{Rb}$ vapor. If necessary, the vapor pressure can be adjusted by heating the $\mathrm{Rb}$ source or by changing the setting of the valve which separates the oven section from the MOT chamber.

The pressure in the BEC chamber is below $10^{-11}$ mbar using a combination of a titanium sublimation (TiSub) pump ${ }^{30}$ and an ion getter pump 27 . At this pressure we achieve lifetimes of the atom cloud of more than 2 min which is sufficient to carry out rf evaporative cooling.

In order to maintain a pressure gradient of $p_{\mathrm{MOT}} / p_{\mathrm{BEC}} \approx$ $10^{2}$, a differential pumping tube is used to separate the MOT and the BEC chamber. Since the size of the atom cloud, which has to be transported through the tube, amounts to a few millimeters, we chose the tube diameter to be $8 \mathrm{~mm}$. Molecular flow calculations then determine the tube length to be $115 \mathrm{~mm}$.

The design of the BEC chamber is shown in Fig.3 and 4 After laser cooled atoms from the MOT are magnetically transported into the BEC chamber, evaporation in a Quadrupole-Ioffe configuration (QUIC) trap ${ }^{31}$ brings the atoms to BEC or close to BEC. Afterwards they are vertically transferred to the ion trap in the science chamber. QUIC traps are typically used in combination with a glass cell, as the Ioffe coils need to be placed quite close to the atoms. In our setup where the BEC chamber is physically connected along the horizontal (to the MOT chamber) and the vertical (to the science chamber) direction, strong shear forces are acting on the chamber walls. For this reason we chose stainless steel instead of glass for the construction of the BEC chamber. To

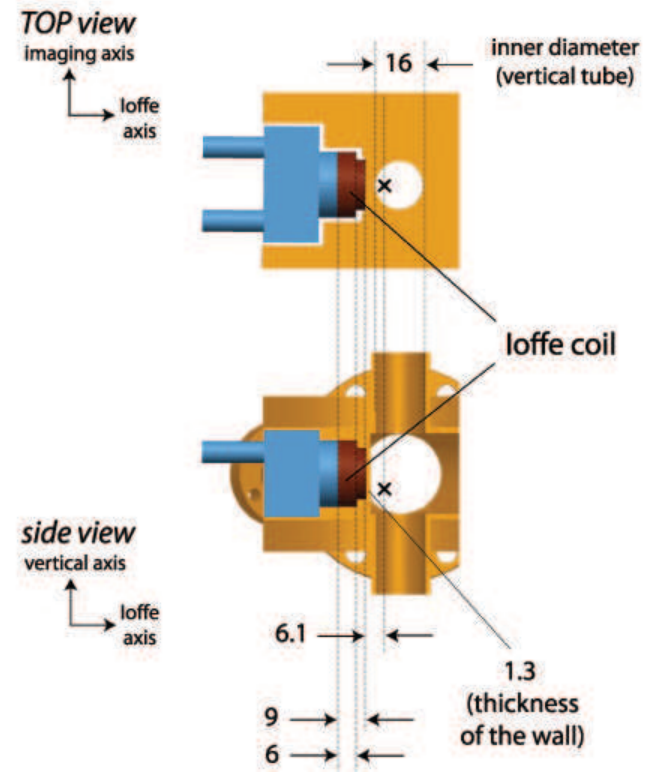

FIG. 4. BEC chamber and Ioffe coil: The BEC chamber features a small insertion slot with an end wall thickness of only $1.3 \mathrm{~mm}$, so that the Ioffe coil (brown) and its holder (blue) can be mounted at a minimum distance of only $11.1 \mathrm{~mm}$ from the center of the chamber. The position of the atom cloud, when it is stored in the QUIC trap, is denoted by the black cross. The dimensions are given in $\mathrm{mm}$.

minimize the distance between the atoms and the Ioffe coil, the BEC chamber exhibits a special insertion slot with a thin end wall into which the Ioffe coil can be placed (see Fig.4). The BEC chamber features four optical axes. The first axis points along the vertical direction and is needed for the optical transport of atoms from the BEC chamber into the science chamber (transport axis). On both ends small DN16CF flanges are used, so that the QUIC quadrupole coils can be easily mounted. The second axis (imaging axis) is used for imaging the ultracold atoms. DN40CF viewports are used on both ends, in order to allow for a good imaging quality. In addition, optical access along the magnetic transport axis is desirable, in order to be able to image the atom cloud at any intermediate position of the transport. Finally, the BEC chamber features a fourth optical axis (optical dipole trap axis), which is currently not used in our experiments, but could for example be employed for the addition of a dipole trapping beam. In order to utilize the full pumping speed of our ion pump, the pumping section is connected to the BEC chamber via a DN100CF flange.

\section{B. Upper section: Science chamber}

The science chamber (Fig.1 and 2), represents the heart of our vacuum apparatus since this is where the experiments take place. It is designed for maximum optical access with eight optical axes. The optical access is needed for cooling and imaging of the ions as well as for trapping, manipulating, and imaging of the atoms. All parts of the ion trap as well as an ob- 


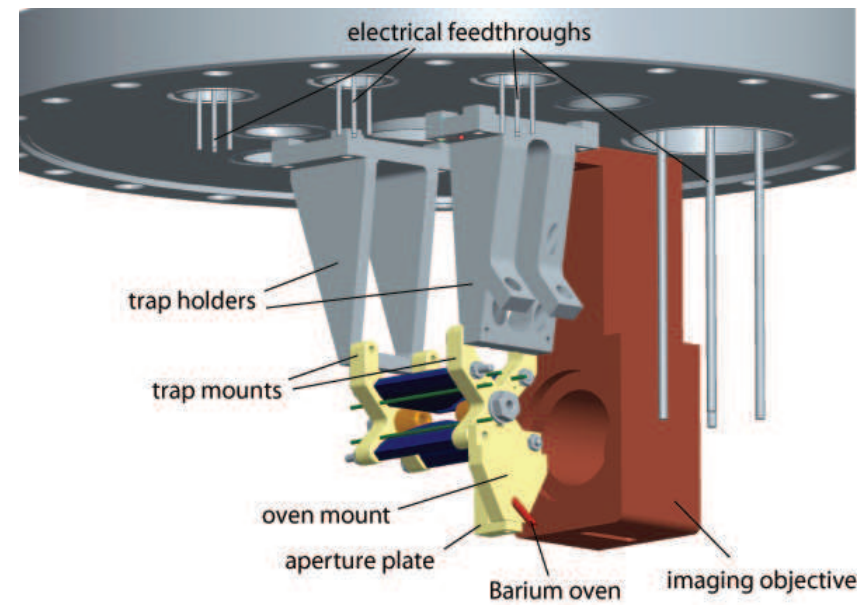

FIG. 5. Science flange (DN200CF): The Paul trap (blue and golden) as well as the Ba oven (red) are mounted on MACOR ceramic parts (pale yellow). The imaging objective consists of four lenses, all of them held in place by a massive aluminum mount (brown) (see also section IIID.

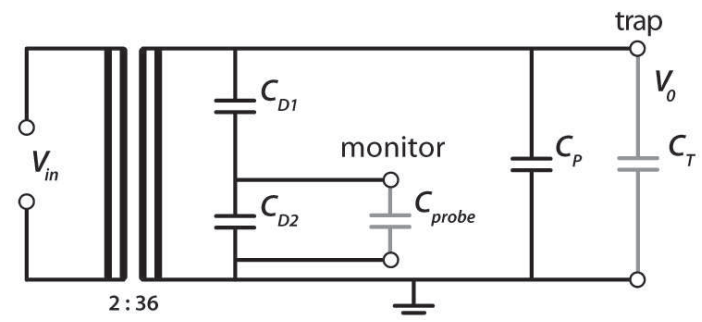

FIG. 6. Ferrite-toroid transformer with a turns ratio of 2:36. By adjusting the capacity $C_{\mathrm{P}}$ the impedance of the trap (corresponding to $C_{\mathrm{T}}$ ) is matched and the ratio between the output and the input voltage $\left(V_{\mathrm{O}} / V_{\text {in }}\right)$ is maximized. In order to be able to monitor the output voltage, a capacitive voltage divider $\left(C_{\mathrm{D} 1}\right.$ and $\left.C_{\mathrm{D} 2}\right)$ is used.

ject lens to collect the ion fluorescence are mounted within the science chamber onto the "science flange" (Fig.5). The flange features various electrical feedthroughs, which are needed to apply the required high voltages to the Paul-trap electrodes and to run currents of up to $12 \mathrm{~A}$ through the $\mathrm{Ba}$ oven. The DN200CF science flange is mounted on top of the science chamber.

The stainless steel, octagon-shaped science chamber (Fig.1) is evacuated by a combination of an ion getter pump and a TiSub pump. As an optional addition of our setup, we have connected a channeltron ion detector ${ }^{32}$ along the axis of the linear ion trap (see also Fig.1 and 2). One possible application of the channeltron is the identification of ions via time-of-flight mass spectrometry as has been demonstrated in other experiments (see for example ${ }^{33}$ ).

\section{ION TRAPPING}

\section{A. Linear Paul trap}

We employ a linear radiofrequency (rf) Paul trap 34 (see Fig.7) to store $\mathrm{Ba}^{+}$or $\mathrm{Rb}^{+}$ions. For the trapping of the ions along the two radial directions we use four blade electrodes, which are mounted symmetrically at a distance of $r_{0}=2.1 \mathrm{~mm}$ from the trap axis. A rf of $\Omega=2 \pi \times 5.24 \mathrm{MHz}$ with an amplitude of $2 \times U_{\mathrm{rf}}=1400 \mathrm{~V}_{\mathrm{pp}}$ is applied to two of the four blades, whereas the other two are grounded. The rf is generated by a commercially available function generator ${ }^{35}$ and subsequently amplified by a $5 \mathrm{~W}$ rf amplifier from Minicircuits 36 . The power at the output of the amplifier is inductively coupled to the trap electrodes via a ferrite-toroid transformer (see Fig.6). The impedance of the trap is matched, so that the supply voltage is resonantly enhanced by a factor of 30 . Trapping along the axial direction is achieved by applying dc voltages on the order of $100 \mathrm{~V}$ to the two endcap electrodes which are located on the trap axis at a distance of $7 \mathrm{~mm}$ from the trap center. Typical ion trapping frequencies for the parameters given above are $\omega_{\text {rad }} \approx 2 \pi \times 250 \mathrm{kHz}$ and $\omega_{\mathrm{ax}} \approx 2 \pi \times 80 \mathrm{kHz}$ for $\mathrm{Ba}^{+}$ and $\omega_{\text {rad }} \approx 2 \pi \times 390 \mathrm{kHz}$ and $\omega_{\mathrm{ax}} \approx 2 \pi \times 100 \mathrm{kHz}$ for $\mathrm{Rb}^{+}$. As expected, the trap frequencies of our ions in the linear Paul trap scale in first order as $\omega_{\text {rad }} \propto 1 / m$ and $\omega_{\text {ax }} \propto \sqrt{1 / m}$. The stability factor $q$ is generally given by $q=2 e U_{\mathrm{rf}} /\left(m \gamma r_{0}^{2} \Omega^{2}\right)^{42}$, where $\gamma=1.53$ is a numerical factor that depends on the exact geometry of the rf electrodes. Our Paul trap allows stable trapping of both, $\mathrm{Ba}^{+}$and $\mathrm{Rb}^{+}$, since $q \ll 1$ for both species $\left(q=0.13\right.$ for $\mathrm{Ba}^{+}$and $q=0.21$ for $\mathrm{Rb}^{+}$).

All electrodes are made of non-magnetic, high-grade, stainless steel of type AISI 316L. This material is specified to have a magnetic permeability of $\mu<1.005$. The blades are produced by electrical discharge machining, which allows for a higher precision and a smaller surface roughness as compared to milling. The electrodes are mounted onto two insulating parts, which are made out of a machinable glass-ceramic (MACOR). This material has a very low outgassing rate and is thus well suited for UHV applications.

\section{B. Loading and laser cooling of ions}

We load the Paul trap (Fig. 7) with either ${ }^{138} \mathrm{Ba}^{+}$or ${ }^{87} \mathrm{Rb}^{+}$ ions. To work with $\mathrm{Ba}^{+}$, we run a current of about $8 \mathrm{~A}$ (corresponding to $6 \mathrm{~W}$ ) through the commercially available $\mathrm{Ba}$ source ${ }^{38}$ (see Fig.5). It is a stainless steel tube with a diameter of $2 \mathrm{~mm}$, which is filled with metal alloy containing $\mathrm{Ba}$. Ba vapor is created through sublimation out of the alloy. In the center of the trap, the neutral $\mathrm{Ba}$ atoms emitted by the oven are photoionized using a diode laser operating at $413 \mathrm{~nm} \cdot \frac{39}{} \mathrm{~A}$ few $\mathrm{mW}$ of laser power are used to drive the resonant two-photon transition from the ground state to the continuum via the ${ }^{3} \mathrm{D}_{1}$ state. With this procedure we are able to load single ions into our trap within a few minutes.

We perform Doppler cooling of the ${ }^{138} \mathrm{Ba}^{+}$ions on the $6 \mathrm{~S}_{1 / 2} \rightarrow 6 \mathrm{P}_{1 / 2}$ cycling transition, which has a transition wavelength of $493 \mathrm{~nm}$ and a linewidth of $15.1 \mathrm{MHz}$. The corre- 


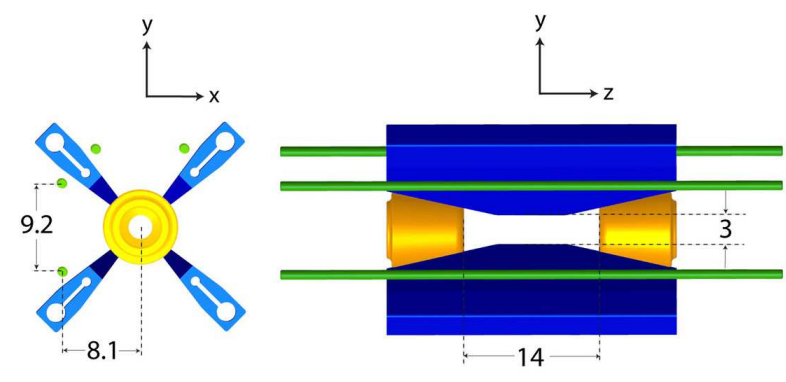

FIG. 7. Linear ion trap (Paul trap). The trap consists of four rf electrodes (blue) for confinement in the x-y plane, two endcap electrodes (golden) for confinement along the $\mathrm{z}$ axis, and four compensation electrodes (green) for the generation of dc electrical fields in the $\mathrm{x}$ y plane. All electrodes are made of stainless steel AISI 316L. The dimensions are given in $\mathrm{mm}$.

sponding Doppler temperature is $360 \mu \mathrm{K}$. The $493 \mathrm{~nm}$ cooling light is generated via frequency doubling of a $986 \mathrm{~nm}$ diode laser. Both the diode laser and the frequency-doubling stage are part of the commercially available system "DL SHG" from Toptica. To stabilize the frequency of the $493 \mathrm{~nm}$ light, the $986 \mathrm{~nm}$ laser is locked to a temperature-stabilized optical cavity using the well-establishd Pound-Drever-Hall method 40.41 . An additional "repumper" laser at a wavelength of $650 \mathrm{~nm}$ is needed, in order to bring the atoms from the metastable $5 \mathrm{D}_{3 / 2}$ state back into the cooling cycle. The repumper is a home-made external-cavity diode laser, which features an anti-reflection-coated laser diode to guarantee stable lasing at the desired wavelength. The frequency of the repumper is stabilized using the same locking scheme as for the $986 \mathrm{~nm}$ laser.

To work with clouds of $\mathrm{Rb}^{+}$ions, we transport ultracold $\mathrm{Rb}$ atoms into the center of the Paul trap and ionize them using the imaging laser at $780 \mathrm{~nm}$ together with the ionization laser at $413 \mathrm{~nm}$. The resonant imaging laser brings the $\mathrm{Rb}$ atom into the $5 \mathrm{P}_{3 / 2}$ state, so that the $413 \mathrm{~nm}$ laser can then drive the transition into the continuum. Since we start with an ultracold trapped atom source, this ionization procedure is very fast and efficient. It allows for loading clouds of $\mathrm{Rb}^{+}$ions into the Paul trap within a few milliseconds.

We employ a different scheme when performing experiments with single $\mathrm{Rb}^{+}$ions. In this case, we first load a single $\mathrm{Ba}^{+}$ion into the Paul trap following the procedure described above. Then we let the $\mathrm{Ba}^{+}$ion interact with ultracold $\mathrm{Rb}$ atoms until the charge transfer process $\mathrm{Rb}+\mathrm{Ba}^{+} \rightarrow \mathrm{Rb}^{+}+\mathrm{Ba}$ has taken place. This takes typically a few seconds. The newly formed $\mathrm{Rb}^{+}$ion is "dark", as it cannot be detected via standard fluorescence imaging due to the lack of an accessible cycling transition. Therefore $\mathrm{Rb}^{+}$has to be detected via its elastic collisions with the neutral atoms and the corresponding atom losses. The newly formed $\mathrm{Rb}^{+}$ion is available for thousands of experimental cycles, since its lifetime in the Paul trap is typically on the order of days.

\section{Micromotion}

In addition to the pseudopotential generated by the rf drive, dc stray electric fields are also present. Possible sources of these fields are imperfections in the fabrication of the trap or patch charges on the ceramic parts. Such surface charges could be generated by our blue $\mathrm{Ba}^{+}$lasers $(413 \mathrm{~nm}$ and $493 \mathrm{~nm}$ ) via the photoelectric effect. In any case, the dc fields push the ion out of its ideal trap location, the rf node, into a region of non-vanishing rf, leading to the so-called "excess micromotion" of the ion. ${ }^{42}$ In order to minimize this enhanced micromotion, we have to compensate the dc electric offset fields at the position of the ion. To compensate electrical offset fields in the axial direction we can apply corresponding voltages to the endcaps of the Paul trap. To generate compensation fields along the radial direction, four "compensation electrodes" (two for each direction) are added to the design of the linear Paul trap. By placing the electrodes at a distance of $9.35 \mathrm{~mm}$ from the trap axis and applying a voltage $U_{\text {comp }}$, we are able to generate dc compensation fields of $E_{\text {comp }}=\beta U_{\text {comp }}$, where $\beta=3.1 \mathrm{~m}^{-1}$.

A simple and in our system very accurate method to detect excess micromotion is to determine the position shift of the ion when the rf amplitude is changed. By adjusting the compensation fields such that the position shift is minimized, we can assure that the potential minimum nearly coincides with the rf node. This method works nicely for electric fields along the vertical direction. However, for the compensation of fields along the $x$-axis a different method has to used, since we are not able to measure the $x$ position of the ion with high accuracy. One possibility, realized in our setup, is to modulate the amplitude of the rf drive. Setting the modulation frequency equal to the trap frequency leads to resonant heating of the ion, which can be detected via a smearing of the ion fluorescence. The heating is particularly strong when the ion is not in the rf node. Hence, we can adjust the compensation fields by minimizing the heating. In early experiments, using the methods described here, we were able to reduce the dc electric fields at the position of the ion to about $1 \mathrm{~V} / \mathrm{m}^{17}$

\section{Imaging methods}

We detect the ion by collecting its fluorescence using a high-aperture laser objective (HALO) from Linos (see Fig. 7). The HALO has a numerical aperture of NA=0.2 and a focal length of $f=60 \mathrm{~mm}$, which enables us to detect about $\mathrm{NA}^{2} / 4 \approx 1 \%$ of the spontaneously emitted photons. It is placed inside the vacuum chamber at a distance of $f=60 \mathrm{~mm}$ from the trap center. Since the original mount of the HALO is anodized and generally not designed to be put into a UHV environment, it was exchanged by a UHV-capable aluminum mount. This new mount features an air vent in order to avoid slow outgassing of air that is enclosed in between the different lenses of the objective.

The collimated fluorescence light exits the chamber through a DN63CF AR-coated viewport. An $f=300 \mathrm{~mm}$ achromat is then used to focus the light onto the EM-CCD chip of an An- 
dor Luca(S) camera. Diffraction at the aperture of the HALO ultimately limits the resolution of the imaging system to about $1.5 \mu \mathrm{m}$, which is an order of magnitude smaller than the typical distance between two neighboring ions of an ion string.

Absorption imaging of the cloud of neutral atoms is also done with the HALO. To separate the $\mathrm{Rb}$ imaging beam $(780 \mathrm{~nm})$ from the $\mathrm{Ba}^{+}$fluorescence light $(493 \mathrm{~nm})$, we use a dichroic mirror. Together with the HALO, an $f=200 \mathrm{~mm}$ achromat forms the objective for the neutral atom detection. The large spacing between the ion-trap electrodes allows for taking the images not only in-situ but also after a free expansion of the atom cloud of up to $15 \mathrm{~ms}$.

\section{PREPARATION AND DELIVERY OF ULTRACOLD ATOMIC SAMPLES}

After the ion has been trapped, we start the production of an ultracold atom cloud in the lower section of the vacuum apparatus. The time needed to create a Rb cloud with $2 \times 10^{6}$ atoms at a temperature of about $1 \mu \mathrm{K}$ is approximately $35 \mathrm{~s}$. Another $5 \mathrm{~s}$ are required to transport the atom cloud into the science chamber and to perform further forced evaporative cooling in an optical dipole trap down to BEC or to cold thermal ensembles with typical temperatures of $100 \mathrm{nK}$.

\section{A. MOT loading and magnetic trap}

To operate the MOT, we have set up two external-cavity diode lasers tuned to the $5^{2} \mathrm{~S}_{1 / 2} \rightarrow 5^{2} \mathrm{P}_{3 / 2}$ transition in ${ }^{87} \mathrm{Rb}$. One of the diode lasers is locked to the $|F=2\rangle \rightarrow\left|F^{\prime}=3\right\rangle$ cycling transition using the modulation transfer spectroscopy technique. 43 The light from this laser is amplified with a tapered amplifier ${ }^{44}$ and sent through a polarization-maintaining (PM) optical fiber. After the fiber the total power of $250 \mathrm{~mW}$ is split up into six beams, all of them having a diameter of $30 \mathrm{~mm}$. Using acousto-optical modulators the cooling light is detuned to about $-3.5 \Gamma$ relative to the cycling transition, where $\Gamma=6 \mathrm{MHz}$ is the transition linewidth. Our second $\mathrm{Rb}$ laser is locked to the $|F=1\rangle \rightarrow\left|F^{\prime}=1\right\rangle /\left|F^{\prime}=2\right\rangle$ cross over line using the frequency modulation (FM) technique. ${ }^{45}$ This repumper laser is used to pump the atoms from the $|F=1\rangle$ groundstate back into the cycling transition. To operate the MOT a total repump power of $6 \times 1.5 \mathrm{~mW}$ is employed. The required magnetic field gradient of $B_{z}^{\prime}=8 \mathrm{G} / \mathrm{cm}$ is generated by running a current of $5 \mathrm{~A}$ through a pair of anti-Helmholtz coils. This setup enables us to load about $3 \times 10^{9}{ }^{87} \mathrm{Rb}$ atoms from the background vapor into the MOT.

By turning off the magnetic field and detuning the MOT cooling beams to about $-8.5 \Gamma$, we perform polarization-gradient cooling for a duration of $10 \mathrm{~ms}$. In this way, the temperature of the atoms is reduced to about $40 \mu \mathrm{K}$. In a next step the atoms are optically pumped into the lowest magnetically trappable state $\left|F=1, m_{F}=-1\right\rangle$ and subsequently loaded into a magnetic quadrupole trap. The trap is generated by running $80 \mathrm{~A}$ through the MOT coils leading to a magnetic field gradient of

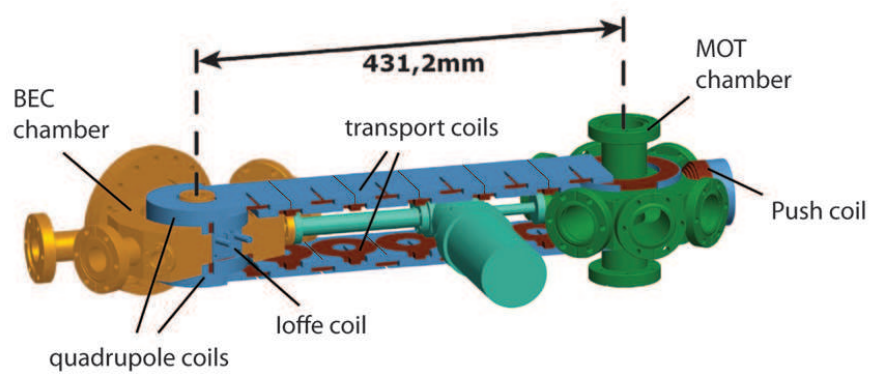

FIG. 8. Magnetic transport line: The neutral atom cloud is transported over a distance of $431.2 \mathrm{~mm}$ from the MOT chamber (green) to the BEC chamber (golden). Together with their respective aluminum housings (blue) the required magnetic field coils (brown) are mounted to the stainless steel chambers.

$B_{z}^{\prime}=130 \mathrm{G} / \mathrm{cm}$, which is sufficient to hold the atoms against gravity. The number of the atoms now amounts to nearly $1 \times 10^{9}$.

\section{B. Magnetic transport and QUIC trap}

Following the concept described in Ref. 46 (see also 47), the cold atom cloud is transported magnetically from the MOT chamber to the BEC chamber. For the transport we employ 13 pairs of anti-Helmholtz coils and an additional "push coil". By properly ramping the currents through the coils, it is possible to smoothly shift the position of the atoms, which are trapped in the magnetic field minimum. We are able to move the cloud over a distance of $431 \mathrm{~mm}$ within $1.5 \mathrm{~s}$. The final particle number after transport is typically $5 \times 10^{8}$, corresponding to an overall transport efficiency of about $50 \%$. The temperature of the atom cloud increases from initially $150 \mu \mathrm{K}$ to about $230 \mu \mathrm{K}$.

At the end of the transport the atoms are loaded into the QUIC trap, which consists of the so-called quadrupole coils together with a small diameter end coil (Ioffe coil) (see Fig.3 and 9). In a first step, the current through the quadrupole coils is ramped from $16 \mathrm{~A}$ (used for the transport) to $36 \mathrm{~A}$ and thus the magnetic field gradient is increased from $B_{z}^{\prime}=130 \mathrm{G} / \mathrm{cm}$ to $B_{z}^{\prime}=320 \mathrm{G} / \mathrm{cm}$. Subsequently, we ramp the current through the Ioffe coil from 0 to $36 \mathrm{~A}$. At the end of the ramp, a single power supply is used to drive the quadrupole coils and the Ioffe coil, which are connected in series. Having all the QUIC coils wired up in the same circuit minimizes heating of the atom sample and leads to a 1/e lifetime of thermal atom clouds of about $2 \mathrm{~min}$. The coil system generates an offset magnetic field of about $2 \mathrm{G}$ and a nearly harmonic potential with trapping frequencies of $\left(\omega_{x}, \omega_{y}, \omega_{z}\right)=2 \pi \times(105,105$, 20) $\mathrm{Hz}$, where the $\mathrm{z}$-direction is along the Ioffe axis.

We perform rf-induced forced evaporative cooling to reduce the temperature of the atom cloud by more than two orders of magnitude. To selectively remove hot atoms, a small coil with 3 turns and a diameter of about $20 \mathrm{~mm}$ is placed inside the vacuum at the bottom of the BEC chamber at a distance of $13 \mathrm{~mm}$ from the atoms. The coil is driven with $30 \mathrm{dBm}$ of $\mathrm{rf}$ power 


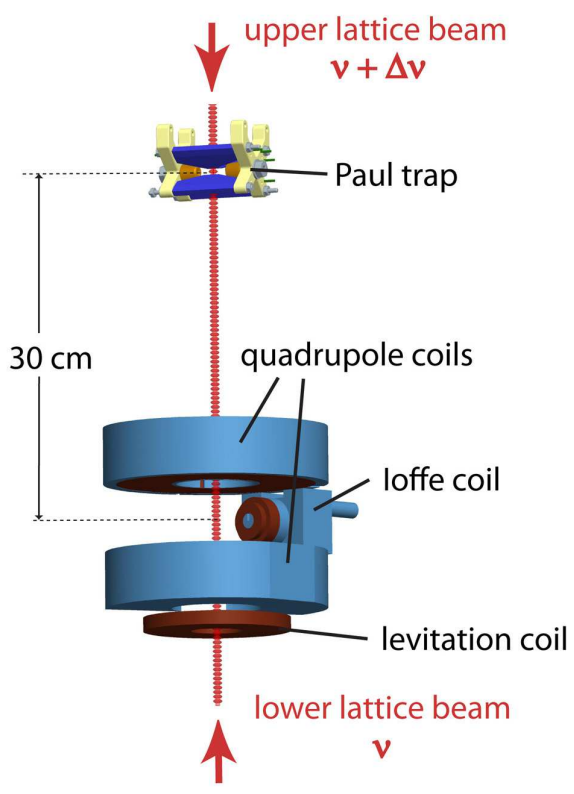

FIG. 9. A moving optical standing wave is used to transport the ultracold atoms vertically from the QUIC trap in the BEC chamber into ion trap in the science chamber. The distance between QUIC trap and Paul trap is not to scale.

and the frequency is ramped from initially $60 \mathrm{MHz}$ down to about $3 \mathrm{MHz}$ within $20 \mathrm{~s}$. With this procedure we are in principle able to produce Bose-Einstein condensates of up to $3 \times 10^{5}$ atoms. However, for our experiments we stop the evaporation before we reach BEC, resulting in a thermal atom cloud with about $1 \mu \mathrm{K}$ temperature and an atom number of about $2 \times 10^{6}$. As compared to the BEC the thermal cloud experiences much smaller losses due to three-body collisions during the subsequent optical transport into the science chamber.

\section{Optical transport of ultracold atoms}

One of the key features of the experimental setup is the vertical long-distance optical transport of the ultracold atoms from the BEC chamber into the science chamber. An illustration is given in Fig.9. We follow a scheme similar to that described in Ref. 48 (where, however, the optical transport was in horizontal direction). The ultracold atoms are first adiabatically loaded from the QUIC trap into a vertical far reddetuned 1-d optical lattice within $300 \mathrm{~ms}$. As the lattice is set into motion it drags along the atoms, like an elevator. After a transport distance of about $30 \mathrm{~cm}$, corresponding to the distance between BEC chamber and science chamber, the lattice is brought to a halt and the atoms are transferred into a crossed dipole trap.

In order to load the atomic cloud into the 1-d optical lattice, it first has to be shifted from its location close to the Ioffe coil (where the evaporation takes place) back to the center of the quadrupole coils which is about $5 \mathrm{~mm}$ away. This shift is controlled via magnetic fields from various magnetic
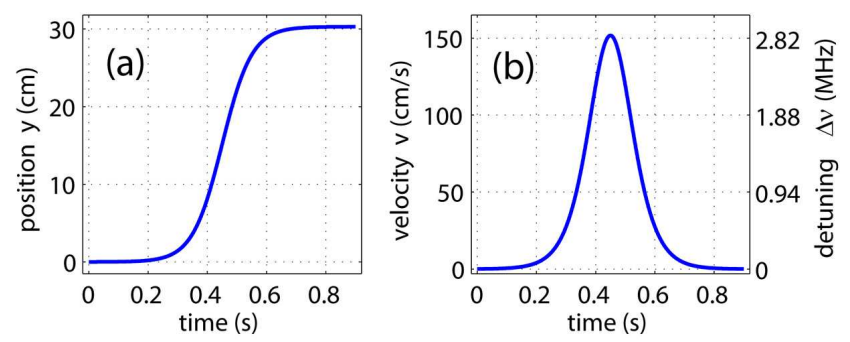

FIG. 10. Optical transport of ultracold atoms. (a) A ramp of the form $y(t)=D[\tanh (n(2 t-T) / T)+\tanh (n)] / 2 \tanh (n)$ is chosen for the vertical position of the atoms versus time, where $D=304 \mathrm{~mm}$ is the transport distance, $T=0.9 \mathrm{~s}$ the transport time, and $n=4.5$ the form parameter. (b) From the ramp $y(t)$ we can derive the velocity $v(t)$ of the atoms and the corresponding relative detuning (frequency shift) $\Delta v(t)$ between the two lattice beams.

coils. Motion along the Ioffe axis (see Fig.3) can be induced by changing the current through the quadrupole coils while keeping the Ioffe current constant. For position changes along the imaging direction (which is orthogonal to the Ioffe axis), we operate the last pair of the magnetic transport coils. The exact position along the (third) vertical axis is irrelevant, since the atoms can be loaded into any antinode of the optical lattice. Nevertheless, we added a levitation coil (see Fig.96) to the system, which can be used to control the vertical position of the atoms. This way we can prevent the atoms from leaving the region to which we have good optical access. In order to optimize the overlap between the magnetic trap and the optical standing wave, we perform Bragg diffraction of the magnetically confined atom cloud using the 1-d optical lattice. We adjust the position of the QUIC trap such that the Bragg diffraction and thus the overlap is maximal. We can determine the lattice depth experienced by the atoms by measuring how the diffraction pattern changes as we vary the length of the Bragg diffraction pulses. For the experimental parameters used in our setup about 100 lattice cells are occupied by the atoms.

The optical lattice is formed by two counterpropagating collimated Gaussian laser beams at $\lambda=1064 \mathrm{~nm}$ with a power of $1.25 \mathrm{~W}$ and $0.5 \mathrm{~W}$, respectively. For both lattice beams, the diameter at the waist was chosen to be about $500 \mu \mathrm{m}$, so that the divergence of the beams can be neglected. The light is derived from a home-made fiber amplifier which is seeded by a diode-pumped solid-state laser. ${ }^{49}$ Due to its very low spectral linewidth $(\approx 1 \mathrm{kHz})$, the laser is well suited for the generation of an optical lattice. Both beams are sent through acoustooptic modulators (AOM) in order to control their frequency as well as their intensity. At the beginning of the transport, both AOMs are driven with a rf of $80 \mathrm{MHz}$. For the transport scheme to work, it is essential that both rfs are phase locked to each other throughout the entire transport sequence because sudden phase jumps would in general lead to atomic loss. Therefore, the rf signals are generated using digital synthesizers (AD9854) which can be locked to the same external reference oscillator. In order to make the standing wave pattern move with a velocity $v=\Delta v \lambda / 2$, we detune the fre- 
quency of the upper lattice beam by $\Delta v$. When the drive frequency of an AOM is modified, the diffraction angle and the beam path of the laser beam changes. To preserve the alignment of the lattice throughout the transport, we couple the upper lattice beam through an optical fiber before sending it to the experiment. The fiber coupling limits the power of the upper lattice beam to about $0.5 \mathrm{~W}$. The lattice beams enter and exit the vacuum system through AR-coated viewports, which are attached to the chamber at an angle of about $4^{\circ}$ with respect to the (vertical) transport axis. By this means we ensure that the reflections off the viewports do not interfere with the standing wave.

Due to the large waist of the laser beams, the confinement of the atoms in the optical lattice sites is more than two orders of magnitude stronger in the axial (transport) direction than in the radial direction. The strong axial confinement prevents gravity from pulling the atoms out of the lattice potential even for moderate laser intensities 50 . Even in the presence of weak heating during transport, the temperature of the atom cloud stays below $1 \mu \mathrm{K}$. This is due to evaporative cooling from the lattice potentials which are only several $k_{\mathrm{B}} \times \mu \mathrm{K}$ deep .

The ramp for the relative frequency shift $\Delta v(t)$ between the two lattice beams is derived from the ramp for the vertical atom position $y(t)$. Both quantities as well as the velocity $v(t)$ are plotted in Fig. 10 as a function of time. During transport, the AOM frequencies and thus also the value for the detuning $\Delta v(t)$ are updated every $40 \mu \mathrm{s}$. For a given transport distance $D$, the transport time $T$ and the form parameter $n$ (see Fig. 10) are optimized for maximum transport efficiency. In our case we have $D=304 \mathrm{~mm}$ and find a maximum efficiency of $60 \%$ for $T=0.9 \mathrm{~s}$ and $n=4.5$, ending up with $1.5 \times 10^{6} \mathrm{Rb}$ atoms in the science chamber.

The optical transport may be extended to even larger distances than described above. As a proof of principle, we have transported the atom cloud from the BEC chamber over $45 \mathrm{~cm}$ to the very top of the science chamber and then back into the BEC chamber again. In this experiment, the total roundtrip distance of $90 \mathrm{~cm}$ was only limited by the length of our vacuum apparatus.

\section{Adjusting the lattice transport distance}

The atom transport has to be adjusted such that it stops at the exact location of the trapped ion. A simple method for this would be to use the same camera to image the locations of the ion and the lattice held atomic cloud and to adjust the transport such that they coincide. However, in our setup this is not possible since we image ions and atoms with two different cameras. We thus use a different approach which proceeds in two steps. First, an approximate value for the transport distance $D$ is found by determining the position of the atom cloud relative to the rf electrodes via standard absorption imaging. We choose $D$ such that the atoms end up roughly in the center of the ion trap midway in between the lower and the upper electrodes.

Second, for a more precise adjustment, we load a cloud of hundreds of ions into the Paul trap. Afterwards we transport

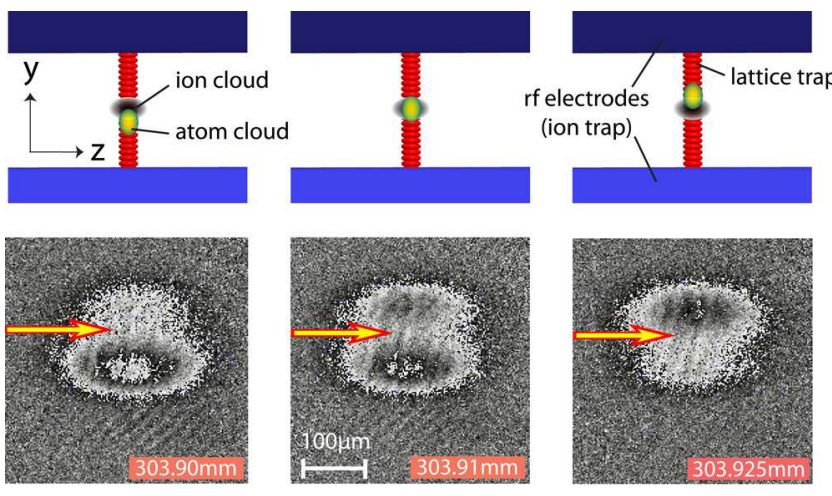

FIG. 11. Absorption images of the atom cloud after the interaction with a localized ion cloud. The atoms are transported to the ion trap and brought into contact with the ion cloud for $1 \mathrm{~s}$. The Paul trap is loaded with a cloud of hundreds of $\mathrm{Rb}^{+}$ions, which are responsible for the localized loss of atoms around the ion trap center. Outside of the ion trap center the atom loss is very small, as the atoms, being trapped in lattice sites, cannot get into contact with the ion cloud. The transport distance is varied between $303.90 \mathrm{~mm}$ to $303.925 \mathrm{~mm}$. Arrows indicate where the ion cloud has depleted the neutral atomic ensemble. For a transport distance of $303.91 \mathrm{~mm}$ the depletion region is located in the center of the atomic cloud, indicating a good vertical alignment. The pictures are taken after a time-of-flight of $12 \mathrm{~ms}$.

a freshly prepared atom cloud to the ion cloud such that the two species get into physical contact. The ion cloud locally depletes the atom cloud which is measured by taking an absorption image of the atom cloud after $1 \mathrm{~s}$ of interaction time (see Fig.11). The depletion is due to elastic collisions between atoms and ions in which atoms simply get kicked out of the shallow lattice potential and are lost. ${ }^{17}$ The loss is local due to the strong confinement in the lattice sites which prevents atoms outside the ion cloud from reaching the ions. The atom cloud typically has an extension on the order of $100 \mu \mathrm{m}$ along the direction of the transport while the ion cloud is well localized within a few tens of $\mu \mathrm{m}$. In order to center the atom cloud on the ion cloud, we adjust the transport distance $D$ such that the location of loss (see arrow in Fig.11) is centered on the atomic cloud. All three images shown in Fig.11 have been taken with the same ion cloud which can be repeatedly used for many depletion experiments.

\section{E. Loading of the crossed dipole trap and evaporative cooling}

After transport, the atoms are loaded into a crossed optical dipole trap, formed by the lower lattice beam and an additional horizontal dipole-trap beam. This additional trapping beam is derived from the same laser as the lattice beams and propagates horizontally along the $x^{\prime}$-axis (see Fig.12), which is at an angle of $45^{\circ}$ with respect to the $\mathrm{x}$-axis. It has a waist of $50 \mu \mathrm{m}$ and is ramped up to a power of $1 \mathrm{~W}$ within $1 \mathrm{~s}$. Subsequently, the power of the upper lattice beam is ramped down to zero within $1 \mathrm{~s}$ and a crossed optical dipole trap is formed. We are able to load about $50 \%$ of the atoms from the 1-d optical 

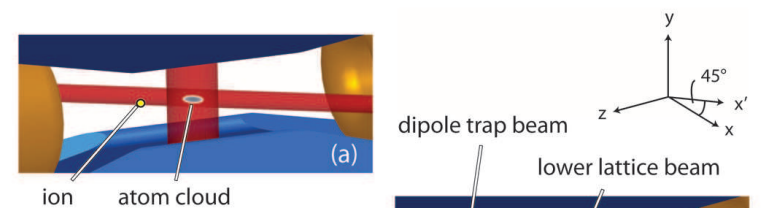

ion atom cloud

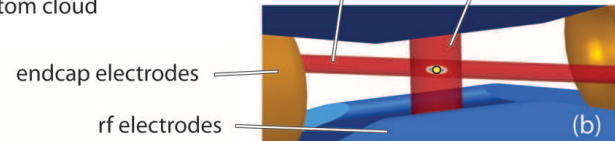

FIG. 12. Probing the position of the optical dipole trap with a single ion. (a) First the ion is moved away $300 \mu \mathrm{m}$ from its normal position to prevent any collisions with the atoms during the final evaporation stage. This is done by changing one of the endcap voltages of the ion trap which moves the ion along the ion-trap axis (z-axis). The position of the optical dipole trap is controlled by moving the laser beams with the help of AOMs. (b) By switching back to the original endcap voltages, the ion is moved back within several ms to its original position where it can now probe the local density of the atomic cloud.

lattice into this crossed dipole trap while keeping the temperature of the sample below $1 \mu \mathrm{K}$.

In the next step, the depth of the dipole trap is lowered within $4 \mathrm{~s}$ to evaporatively cool the atoms and to end up with a BEC of typically $10^{5}$ atoms. If we prefer to work with a thermal atom cloud $(T=100 \mathrm{nK})$, the evaporation is stopped immediately before the onset of Bose-Einstein condensation. The lifetime of a thermal atom cloud is typically on the order of $10^{2} \mathrm{~s}$.

\section{F. Fine alignment of the crossed dipole trap}

We control the position of the crossed dipole trap dynamically with the help of AOMs (see Fig.12). For a typical AOM with a center frequency of $80 \mathrm{MHz}$ the corresponding Bragg angle is about $10 \mathrm{mrad}$. Since the frequency bandwidth is on the order of $10 \%$, the diffraction angle can be varied by about $1 \mathrm{mrad}$. For distances between the AOMs and the science chamber on the order of $1 \mathrm{~m}$, this results in a shift of the ion trap position by up to $1 \mathrm{~mm}$.

The relative position of the dipole trap relative to the ion trap can be accurately measured by again looking at atomic collisional losses as previously described in section IVD. However, this time we use a single ion (see Fig. (12). In order to precisely control the interaction time of the ion with the atomic cloud, the ion is at first positioned about $300 \mu \mathrm{m}$ away from its proper location so that it cannot interact with the atoms. This is done by changing one of the endcap voltages of the linear ion trap. This moves the ion along the axis of the ion trap (z-axis). Afterwards, the atom cloud is transported in from the BEC chamber and positioned by smoothly ramping the AOM frequencies. By quickly switching back the endcap voltages to their proper values, the ion moves back to its original position within $2 \mathrm{~ms}$ and starts colliding with the atoms, kicking them out of the trap. This procedure is illustrated in Fig.12 We then measure the atom loss as a func-

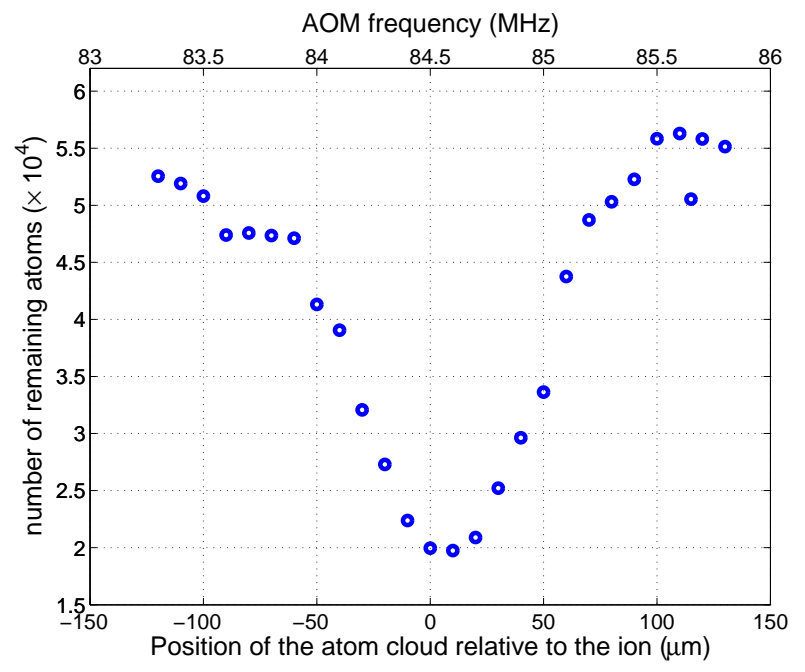

FIG. 13. The number of remaining atoms after a given atom-ion interaction time of $t=1 \mathrm{~s}$. When the center of the atom cloud (i.e. the dipole trap) coincides with the position of the ion the number of remaining atoms is minimal (see also Ref. 17. The position of the dipole trap is controlled by the drive frequency of AOMs. (Here the vertical beam of the crossed dipole trap is moved along the $\mathrm{x}$ 'direction.)

tion of the dipole trap position (i.e. the AOM frequencies). A typical curve is shown in Fig.13 for an interaction time of about $1 \mathrm{~s}$, working with a thermal cloud of initially $5.5 \times 10^{4}$ atoms. Here, the same ion is used for all data points. (The micromotion of the ion was compensated to about $10 \mathrm{mK}$ for these measurements.) The atom number reaches a minimum when the dipole trap is centered onto the ion. We can use this measurement to accurately overlap atom and ion trap along all three directions in space.

\section{SUMMARY}

In conclusion, we have designed a hybrid vacuum apparatus which allows for cold collision experiments between ultracold neutral atoms and trapped, cold, single ions. A main feature of this apparatus is the spatial separation of production of the ultracold atomic cloud and the location where experiments take place, i.e. the linear ion trap. In order to transport the atoms to the ion trap, we use a novel efficient optical elevator consisting of a moving optical lattice which bridges a vertical gap of $30 \mathrm{~cm}$ within $1.5 \mathrm{~s}$. The use of a vertical transport also leads to a non-standard design of the BEC apparatus which houses a QUIC trap within a steel chamber. The overall layout of the apparatus is quite modular which has advantages in terms of stability and versatility, such that extensions can be added to the apparatus easily in the future. Also it helped to optimize the optical access to the region where ion-atom collision experiments take place. In addition to discussing the overall design we also describe various alignment and optimization procedures, e.g. accurate positioning of the atomic cloud onto the ion trap. First experiments with the apparatus 17 
show promise for exciting research prospects in the future.

We would like to thank Rudi Grimm for generous support during the built-up phase. We are grateful to Michael Drewsen and the group of Rainer Blatt for advice on the design of the ion trap. We thank Wolfgang Limmer for thorough proofreading of the manuscript and help in editing. We thank Dennis Huss, Artjom Krükow, Andreas Brunner and Wolfgang Schnitzler for several technical improvements of the set-up. This work was supported by the Austrian Science Foundation (FWF) and the DFG within the SFB/ TR21. S.S. acknowledges support from the Austrian Academy of Sciences within the DOC doctorial research fellowship program.

${ }^{1}$ D. Leibfried, R. Blatt, C. Monroe, and D. Wineland, Rev. Mod. Phys. 75, 281 (2003).

${ }^{2}$ Nature Insight on Ultracold Matter, Nature 416, 205-246 (2002).

${ }^{3}$ M. Hawley, and M. A. Smith, J. Chem. Phys. 95, 8662 (1991).

${ }^{4}$ D. Gerlich, E. Herbst, and E. Roue, Planetary and Space Science 50, 1275 (2002).

${ }^{5}$ D. Gerlich, Phys. Chem. Chem. Phys. 7, 1583 (2005).

${ }^{6}$ R. Otto, J. Mikosch, S. Trippel, M. Weidemller, and R. Wester Phys. Rev. Lett. 101, 063201 (2008).

${ }^{7}$ S. Willitsch, M. T. Bell, A. D. Gingell, S. R. Procter, and T. P. Softley, Phys. Rev. Lett. 100, 043203 (2008).

${ }^{8}$ M. Cetina, A. Grier, J. Campbell, I. Chuang, and V. Vuletic, Phys. Rev. A 76, 041401 (2007).

${ }^{9}$ A. T. Grier, M. Cetina, F. Orucevic, and V. Vuletic, Phys. Rev. Lett. 102, 223201 (2009).

${ }^{10}$ F. H. J. Hall, M. Aymar, N. Bouloufa-Maafa, O. Dulieu, and S. Willitsch, Phys. Rev. Lett. 107, 243202 (2011).

${ }^{11}$ W. G. Rellergert, S. T. Sullivan, S. Kotochigova, A. Petrov, K. Chen, S. J. Schowalter, and E. R. Hudson, Phys. Rev. Lett. 107, 243201 (2011).

${ }^{12}$ K. Ravi, S. Lee, A. Sharma, G. Werth, S.A. Rangwala, Appl. Phys. B: Lasers and Optics, DOI 10.1007/s00340-011-4726-6 (2011).

${ }^{13} \mathrm{M}$. Weidemüller and Roland Wester, private communication.

${ }^{14}$ C. Zipkes, S. Palzer, C. Sias, and M. Köhl, Nature 464, 388-391 (2010).

${ }^{15}$ C. Zipkes, S. Palzer, L. Ratschbacher, C. Sias, and M. Köhl, Phys. Rev. Lett. 105, 133201 (2010).

${ }^{16}$ C. Zipkes, L. Ratschbacher, S. Palzer, C. Sias, and M. Köhl, J. Phys.: Conf. Ser. 264, 012019 (2011)

${ }^{17} \mathrm{~S}$. Schmid, A. Härter, and J. Hecker Denschlag, Phys. Rev. Lett. 105, 133202 (2010).

${ }^{18}$ R. Côté, and A Dalgarno, Phys. Rev. A 62, 012709 (2000).

${ }^{19}$ Z. Idziaszek, T. Calarco, P. S. Julienne, and A. Simoni, Phys. Rev. A 79, 010702 (2009).
${ }^{20}$ P. F. Staanum, K. Hojbjerre, P. Skyt, A. Hansen, and M. Drewsen, Nat. Phys. 6, 271 (2010).

${ }^{21}$ T. Schneider, B. Roth, H. Duncker, I. Ernsting, and S. Schiller, Nat. Phys. 6, 275 (2010).

${ }^{22}$ P. Massignan, C. J. Pethick, and H. Smith, Phys. Rev. A 71, 023606 (2005).

${ }^{23}$ R. M. Kalas, and D. Blume, Phys. Rev. A 73, 043608 (2006).

${ }^{24}$ F. M. Cucchietti, and E. Timmermans, Phys. Rev. Lett. 96, 210401 (2006).

${ }^{25}$ R. Côté, Phys. Rev. Lett. 85, 5316 (2000).

${ }^{26}$ R. Côté, V. Kharchenko, and M. D. Lukin, Phys. Rev. Lett. 89, 093001 (2002).

${ }^{27}$ Varian StarCell 751/s.

${ }^{28}$ Varian UHV-24p.

${ }^{29}$ Sigma-Aldrich Part-No 276332-1G.

${ }^{30}$ Titanium Sublimation pump TSP (filament type) from Varian.

${ }^{31}$ T. Esslinger, I. Bloch, and T. W. Hänsch, Phys. Rev. A 58, R2664-R2667 (1998).

${ }^{32}$ CEM-4823G from Burle.

${ }^{33}$ K. Ravi, S. Lee, A. Sharma, G. Werth, and S. A. Rangwala, arXiv: $1009.4515 \mathrm{v} 3$ (2011).

${ }^{34}$ M. Raizen, J. M. Gilligan, J. C. Bergquist, W. M. Itano, and D. J. Wineland, Phys. Rev. A 45, 6493 (1992).

${ }^{35}$ Agilent 33220A.

${ }^{36}$ Minicircuits ZHL-5W-1.

${ }^{42}$ D. J. Berkeland, J. D. Miller, J. C. Bergquist, W. M. Itano, and D. J. Wineland, J. App. Phys. 83, 10 (1998).

${ }^{38}$ Alvasource from the company Alvatec.

${ }^{39}$ DL-100 from Toptica.

${ }^{40}$ R. W. P. Drever, J. L. Hall, F. V. Kowalski, J. Hough, G. M. Ford, A. J. Munley und H. Ward, Appl. Phys. B 31, 97-105 (1983).

${ }^{41}$ E. D. Black, Am. J. Phys. 69, 79 (2001).

${ }^{42}$ D. J. Berkeland, J. D. Miller, J. C. Bergquist, W. M. Itano, D. J. Wineland, J. Appl. Phys. 83, 5025 (1998).

${ }^{43}$ D. J. McCarron, S. A. King, and S. L. Cornish, Meas. Sci. Technol. 19, 105601 (2008).

${ }^{44}$ BoosTA from Toptica.

${ }^{45}$ G. C. Bjorklund, M. D. Levenson, W. Lenth, and C. Ortiz, Appl. Phys. B 32, 145-152 (1983).

${ }^{46}$ M. Greiner, I. Bloch, T. W. Hänsch, and T. Esslinger, Phys. Rev. A 63, 031401 (2001).

${ }^{47}$ M. Theis, G. Thalhammer, K. Winkler, M. Hellwig, G. Ruff, R. Grimm, and J. Hecker Denschlag 93, 123001 (2004).

${ }^{48}$ S. Schmid, G. Thalhammer, K. Winkler, F. Lang, and J. Hecker Denschlag, New. J. Phys. 8, 159 (2006).

${ }^{49}$ Mephisto from the company Innolight.

${ }^{50}$ This is in fact the main reason for the vacuum chamber layout where the position of the science chamber is located above the BEC chamber. A horizontal transfer into the science chamber would have been plagued with compensating the gravitational force to prevent atomic losses. 